Supporting Information

\title{
Individual Electron and Hole Mobilities in Lead Halide Perovskites Revealed by Noncontact Methods
}

Yaxin Zhai, Kang Wang, Fei Zhang, Chuanxiao Xiao, Aaron Rose, Kai Zhu, and Matthew C. Beard* $^{*}$

Chemical and Nano Science Center, National Renewable Energy Laboratory, Golden, Colorado 80401, United States

\section{Corresponding Author}

*Matthew C. Beard, Matt.Beard@nrel.gov.

\section{Experimental Section}

Sample Preparation:

The lead halide perovskites precursor solution was prepared in a glovebox from a $1.35 \mathrm{M} \mathrm{Pb}^{2+}$ $\left(\mathrm{PbI}_{2}\right.$ for $\mathrm{MAPbI}_{3} ; \quad \mathrm{PbI}_{2}$ and $\mathrm{PbBr}_{2}$ for $(\mathrm{FAI})_{0.85}\left(\mathrm{PbI}_{2}\right)_{0.85}(\mathrm{MABr})_{0.15}\left(\mathrm{PbBr}_{2}\right)_{0.15}$ and $\left.\left(\mathrm{CsPb}_{3}\right)_{0.05}\left(\mathrm{FAPbI}_{3}\right)_{0.85}\left(\mathrm{MAPbBr}_{3}\right)_{0.15}\right)$ in the mixed solvent of Dimethylformamide (DMF) and Dimethyl sulfoxide (DMSO); the volume ratio of DMF/DMSO is 4:1. The spin-coating procedure was performed by $2,000 \mathrm{rpm}$ for $10 \mathrm{~s}$ followed with $6,000 \mathrm{rpm}$ for $30 \mathrm{~s}$. At $15 \mathrm{~s}$ before the last spin-coating step, $140 \mu \mathrm{L}$ of chlorobenzene was pipetted onto the $1 \mathrm{~cm} \times 2 \mathrm{~cm}$ quartz substrate. Thereafter, the samples were baked on a hotplate for 1 hour at $100^{\circ} \mathrm{C}$.

\section{Ellipsometry Spectroscopy}

Ellipsometry data was taken at incident angles of $60^{\circ}, 65^{\circ}$, and $70^{\circ}$ on each film using a J. A. Woollam M-2000DI ellipsometer and was modeled with transmittance data in CompleteEASE 6.34 by J. A. Woollam. The model consisted of the thin perovskite film on quartz and was used to 
extract the complex refractive index and thickness of each film. The optical properties of a blank quartz substrate were measured and modeled using the Cauchy method. The refractive indices of the perovskites were fit to the sum of a Drude term, a parametric semiconductor oscillator ('PSemiM0'), and five Lorentzian distributions. To properly account for surface effects, the model divided the film into 11 slices and three nodes, allowing the Drude parameters and the PSemi-M0 amplitude to vary linearly from the surface nodes to a variable-position central node. The Drude term accounts for free carriers while the PSemi-M0 term is a flexible Kramers-Kronig consistent shape used to fit the band edge. The fitting routine uses the least squares method and for each film attained a root mean square squared error of less than 10 .

\section{Time-Resolved THz Spectroscopy (TRTS)}

The TRTS measurements were performed by a pump-probe spectrometer. The fundamental laser pulse with wavelength at $800 \mathrm{~nm}$ is generated by a Ti:sapphire amplifier. The pulse repetition rate is $1 \mathrm{kHz}$. The fundamental pulse is then split into two parts by a beam splitter. One part is sent to an optical parametric amplifier for the pump generation at $2.48 \mathrm{eV}$. The pump is chopped at a frequency of $500 \mathrm{~Hz}$ and attenuated by neutral density filter wheels. The average excitation density is calculated as the ratio of input photon flux to the pump penetration depth. The probe beam has two arms. The first arm generates freely propagating $\mathrm{THz}$ pulses that are focused onto a sample, transmitted, and refocused onto a detector crystal. It is overlapped spatially and temporally with the optical pump pulse at the sample position; the delay between the THz pulse and the pump pulse is controlled by a delay stage. The second arm of probe beam controls a gating near-IR pulse for free space electro optic sampling of the $\mathrm{THz}$ pulses. The gating pulse and the transmitted $\mathrm{THz}$ pulse are overlapped spatially and temporally at the detector crystal.

\section{Transient Reflection (TR) Spectroscopy}

The TR shared the same laser source as TRTS, and the fundamental pulse is then split into two parts by a beam splitter. The pump part is set to be same condition as TRTS to ensure same carrier density for the two measurements. The other part of the fundamental pulse is focused into a sapphire crystal to generate a white-light continuum $(1.55 \mathrm{eV}$ $2.7 \mathrm{eV}$ ) that is used as the probe. The probe pulses are delayed in time with respect to the 
pump pulses using a motorized translation stage mounted with a retroreflecting mirror. The pump and probe are spatially overlapped on the surface of the sample. The incident angle for probe is around $45^{\circ}$, while the pump beam incident the sample normally. The size of the focused spot at the sample position for the probe and pump beams is around $200 \mu \mathrm{m}$ and $600 \mu \mathrm{m}$, respectively.

\section{Nanosecond Transient Absorption (TA) Spectroscopy in Nanosecond Scale}

Nanosecond transient absorption spectra were collected using a EOS systems and a Coherent Libra Ti:sapphire laser, with an output of $800 \mathrm{~nm}$ at $1 \mathrm{kHz}$. The $800 \mathrm{~nm}$ beam was directed into a TOPAS optical parametric amplifier to generate pump pulse $(\sim 150 \mathrm{fs})$ and was modulated at $500 \mathrm{~Hz}$ through an optical chopper to block every other laser pulse. The broadband EOS probe beam was electronically delayed respect to pump laser pulse.

\section{Kelvin Probe Force Microscopy (KPFM)}

KPFM measurements were performed on a Veeco D5000 atomic force microscope (AFM) equipped with a Nanoscope V controller. The AFM system operates in an Ar-filled glovebox with water and oxygen level $<0.1 \mathrm{ppm}$. KPFM measures the contact potential difference between the probe (Nanosensor PPP-EFM, Pt/Ir coated) and sample. In this set-up, the first resonant oscillation of the cantilever $(50-70 \mathrm{kHz})$ was used for the non-contact AFM topographic imaging. The second resonant frequency $(300-500 \mathrm{kHz})$ was used for the potential imaging, which provides an enhanced energy resolution of $\sim 10 \mathrm{mV}$ in the atmospheric pressure. The image of the Kelvin probe signal represents the relative scale of the electrostatic potential on the sample surface. We scanned an area of $2 \mu \mathrm{m} \times 2 \mu \mathrm{m}$ that has 1,024 points on the fast axis and 256 lines on the slow axis with a scan rate of $0.2 \mathrm{~Hz}$. All results were scanned by the same probe; and we scan on at least two locations to make sure that the probe has no change and the results are reliable.

Scanning electron microscopy (SEM)

SEM images were taken by a Hitachi S4800 equipment. The electron beam condition is $3 \mathrm{kV}, 8 \mu \mathrm{A}$.

\section{Individual electron and hole mobilities in intrinsic GaAs wafer}


We first verified our method on a semi-insulating GaAs wafer (AXS). In the transient reflection (TR) spectroscopy, the pump was chosen at $1.55 \mathrm{eV}, 1.91 \mathrm{eV}$ and $2.48 \mathrm{eV}$ as shown in Figure S1(a). In case of the GaAs wafer that the thickness, $L_{G a A s}$ is orders of magnitude thicker than the optical penetration depth plus carrier diffusion length, the boundary conditions are described as

$$
\left.\frac{\partial N(x, t)}{\partial t}\right|_{x=0}=\frac{S}{D} N(0, t), N\left(L_{G a A s}, t\right)=0
$$

where $\mathrm{S}$ is the surface recombination velocity (SRV) and D is the diffusion coefficient. Assuming $\mathrm{L}_{\mathrm{GaAs}}$ is infinitely long, Equation (1) in the main text with initial and boundary conditions for the GaAs wafer can be solved analytically, giving an analytical expression of $N(x, t)$, see reference 12 in the main text. Thus, $\mathrm{S}$ and D can be obtained by simultaneously modeling the different surface carrier kinetics within a global fitting procedure. The surface of the GaAs is not treated, a reasonable large $\mathrm{SRV}=(1.15 \pm 0.03) \times 106 \mathrm{~m} / \mathrm{s}$ is obtained. By using the diffusion coefficient, $\mathrm{D}=9.93 \pm 0.90 \mathrm{~cm} 2 / \mathrm{s}$ at $\mathrm{n}=4.5 \times 1017 \mathrm{~cm}-3$, the ambipolar mobility can be calculated as following,

$$
\frac{1}{\mu_{n}}+\frac{1}{\mu_{p}}=\frac{1}{191 \pm 17 \mathrm{~cm}^{2}(\mathrm{Vs})^{-1}}
$$

In the TRTS measurement, the photoconductivity of GaAs is proportional to photoinduced change ratio in terahertz transmission $\left(\frac{\Delta \tilde{E}}{\tilde{E}_{0}}\right)$ through the following relationship (for small $\frac{\Delta \tilde{E}}{\tilde{E}_{0}}$ ),

$$
\Delta \tilde{\sigma}(t)=-\epsilon_{0} \frac{c}{d}\left(1+n_{G a A s}\right) \frac{\Delta \tilde{E}}{\tilde{E}_{0}}
$$

where $\epsilon_{0}$ is the permittivity of free space, $c$ is the speed of light, $d$ is the penetration depth of the pump beam at corresponding wavelength $(d=14.8 \mathrm{~nm})$ and $\tilde{n}_{G a A s}$ is the refractive index of the GaAs $\left(n_{\text {GaAs }}=3.6\right.$ at $\left.1 \mathrm{THz}\right)$. By modeling the mobility by the simple Drude model we get a DCsum mobility $\Sigma \mu_{D C}$ as indicated in Figure S1(b).

$$
\begin{gathered}
\Sigma \tilde{\mu}=\Sigma \mu_{D C} \frac{1}{1-\frac{i \omega}{\Gamma}} \\
\Sigma \mu_{D C}=3006 \pm 78 \mathrm{~cm}^{2}(\mathrm{Vs})^{-1}
\end{gathered}
$$

The decay dynamics of the real part of photoconductivity at different optical pump fluence are shown in Figure S1(c). Mobility as a function of carrier concentration $\mathrm{N}$ follows the empirical 
Caughey-Thomas curve line $\mu=\mu_{\min }+\frac{\mu_{\max }-\mu_{\min }}{1+\left(\frac{N}{N_{r e f}}\right)^{\alpha}}$, Figure S1(d). Combining Equation (S2) and (S5), the individual carrier mobilities are determined as $\mu_{\mathrm{n}}=2801 \pm 249 \mathrm{~cm}^{2}(\mathrm{Vs})^{-1}$ and $\mu_{\mathrm{p}}=$ $205 \pm 18 \mathrm{~cm}^{2}(\mathrm{Vs})^{-1}$, which agree as well with the average reported values at such carrier density.

\section{Transient reflection (TR) spectroscopy}

In halide perovskite semiconductors at low excited carrier density, the small TR spectrum is approximately proportional to the change of the real part of the refractive index, $\Delta n(\omega)$ because the imaginary part $(k)$ is trivial comparing to $n$ near the band edge, see Figure S2. ${ }^{36}$ On the other hand, the TA spectrum provides the information of $\Delta k(\omega)=\frac{\Delta \alpha(\omega) c}{2 \omega}$. Since $\Delta n(\omega)$ and $\Delta k(\omega)$ are related with the Kramers-Kronig relationship, the Hilbert transform (HT) of TA can represent the TR spectrum, the inverse Hilbert transform (iHT) of TR can represent the TA spectrum. ${ }^{24,46}$ We have performed TR measurement at various pump photon energy for all the three perovskites, and Figure S6 show pseudo-color image of the TR spectra and the spectrum captured at 5 ps for MA polycrystalline film pumping at $2.07 \mathrm{eV}$. In accordance, we find that a linearly scaled HT of TA matches the TR spectrum, indicating that the main TR features arise from ground-state bleaching that is caused by phase-space filling in the presence of free photocarriers. We note that the HT of TA is not overlapped with TR spectrum at low energy. This is caused by the interference fringes from the probe light reflected from both the front and back surface of the thin film that needed to be deconvolved carefully from the total reflectance spectrum. ${ }^{24}$ 

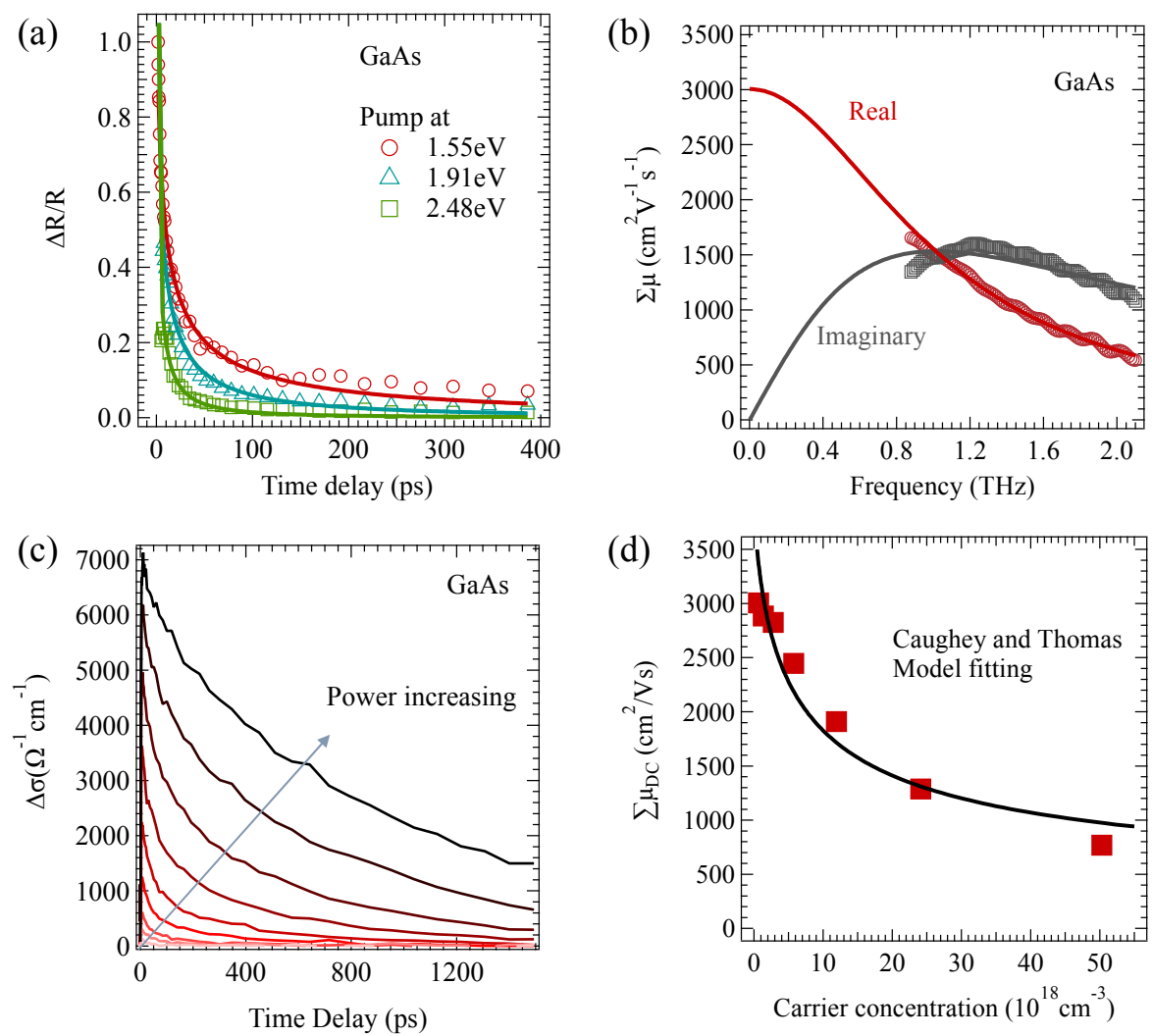

Figure S1. Transient reflection and $\mathrm{THz}$ spectra in semi-insulator GaAs. (a)Surface carrier dynamics measured by TR. (b) Drude model fitting of the sum mobility in GaAs. (c) Power dependence of photoconductivity. (d) The sum of DC conductivity versus carrier concentration. The Caughey and Thomas model fitting applied.
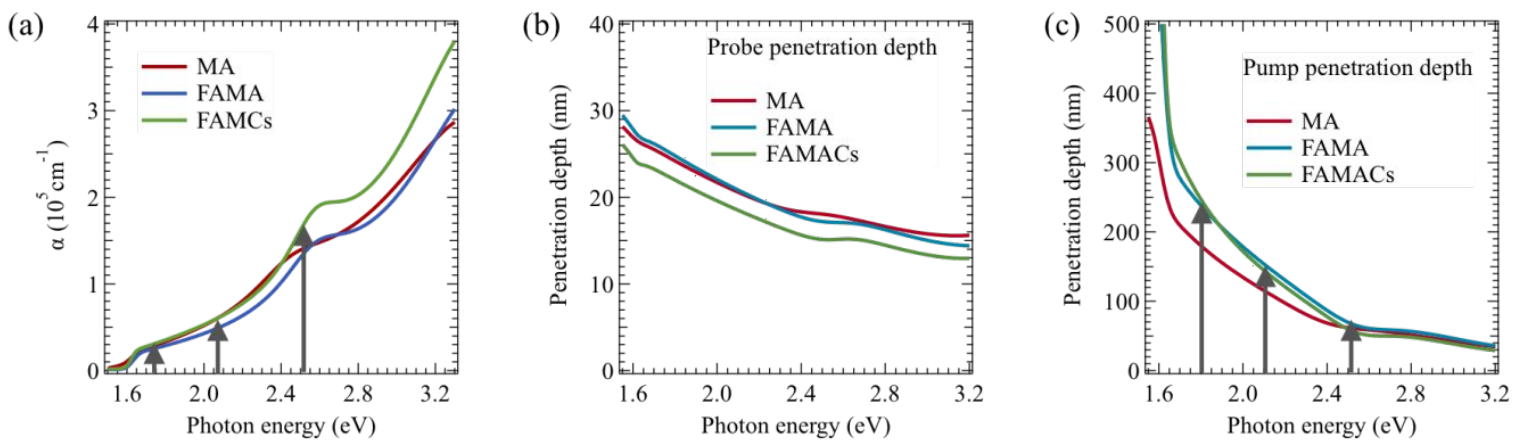

Figure S2. (a) Absorption coefficient $\alpha(\hbar \omega)$ determined from the imaginary part of the complex refractive indices as a function of photon energy. (b) Penetration depth of the probe beam in transient reflection spectroscopy derived by $d(\hbar \omega)=\frac{\lambda}{4 \pi n(\hbar \omega)}$. (c) Penetration depth of the pump beam in transient reflection spectroscopy derived by $d(\hbar \omega)=\frac{\lambda}{4 \pi k(\hbar \omega)}$. 


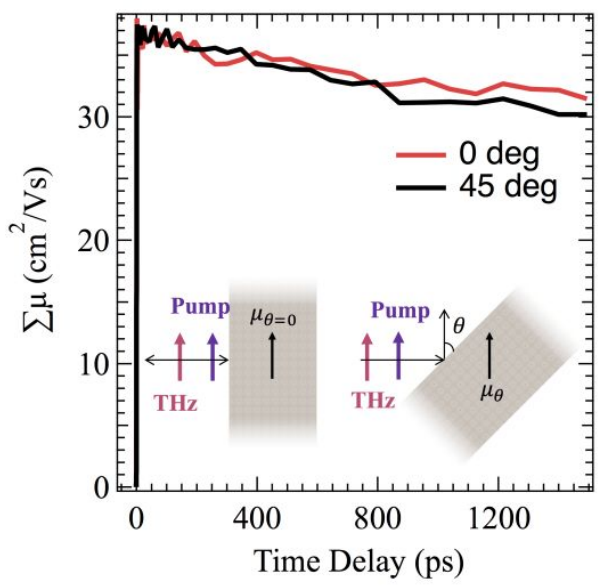

Figure S3. Sum mobility of the MAPI perovskite at both normal and tilted incident angles.
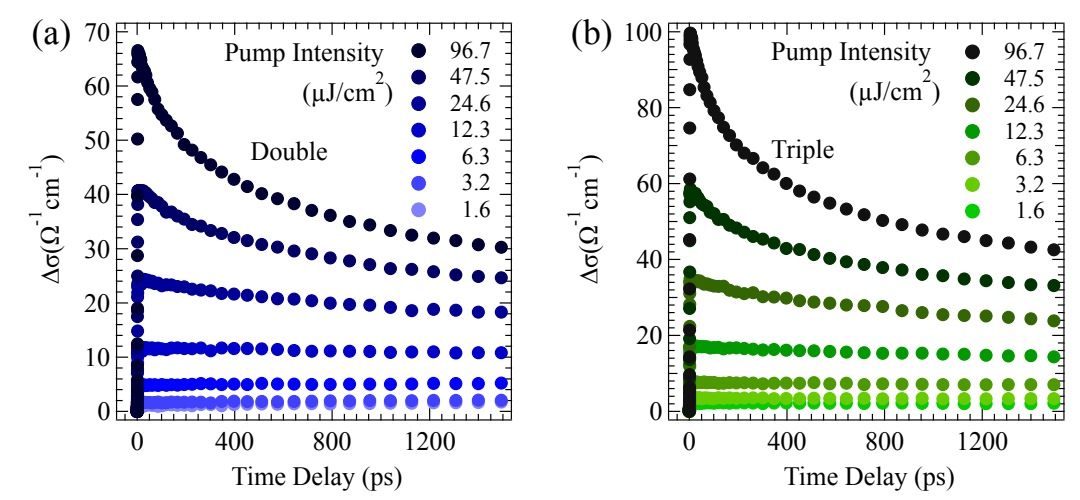

Figure S4. Decay kinetics of the real part of photo-conductivity, $\Delta \sigma_{1}(t)$ measured at the peak of the timedomain THz spectrum with different power density in (a) FAMA and (b) FAMACs films.
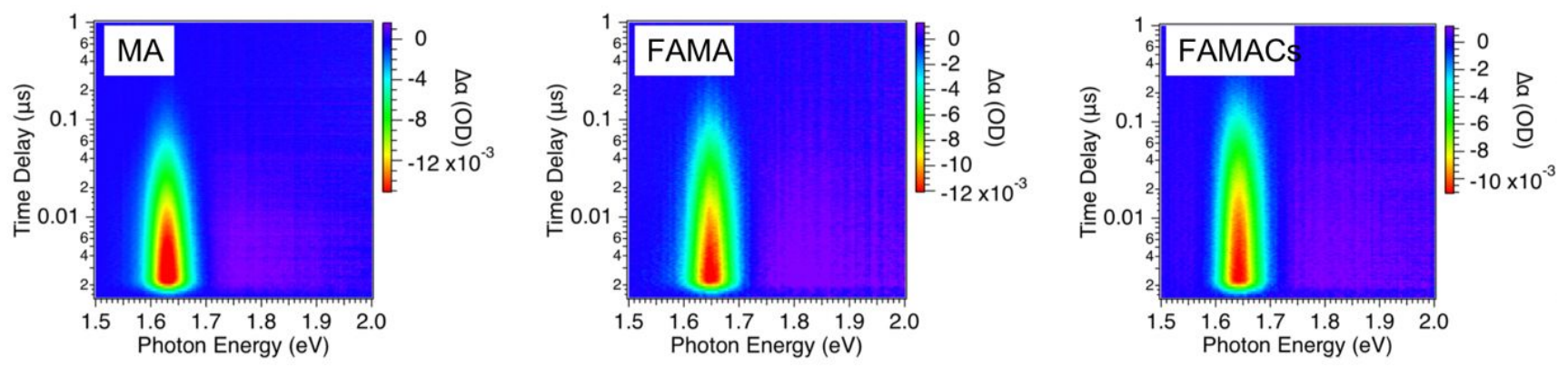

Figure S5. Transient absorption spectra in nanosecond scale of MA, FAMA and FAMACs. The total carrier life time of each film are calculated in Table S1. 

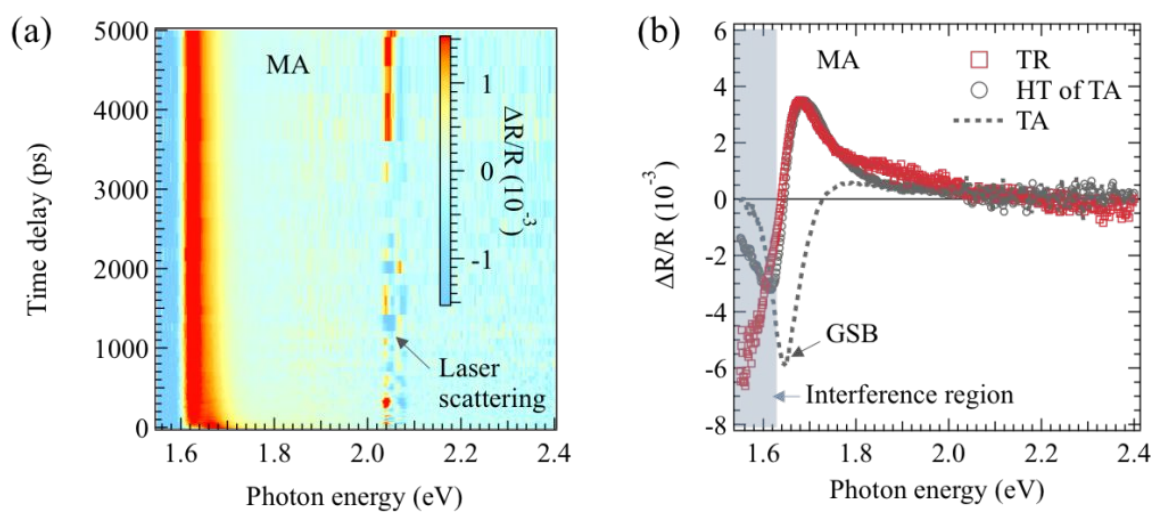

Figure S6. (a) Pseudo-color image of the TR spectra for MA polycrystalline film. The horizontal and vertical axes are the probe-photon energy and pump-probe delay, respectively. The color intensity indicated by the scale bar represents the TR signal magnitude. The noise at $2.07 \mathrm{eV}$ is due to the pump laser scattering. (b) TR (red squares), TA (dashed line) and linearly scaled Hilbert transform (HT) of the TA (gray circles) spectra at $\mathrm{t}=10 \mathrm{ps}$ for MA films. The HT of the TA spectrum obtained from the Kramers-Kronig relationship is consistent with the TR spectrum above $1.7 \mathrm{eV}$, suggesting the TR spectrum in the overlapping region is caused exclusively by the change in reflectance at the front surface. The spectral features in the grey shaded region result from the interference in the thin film.

(a)

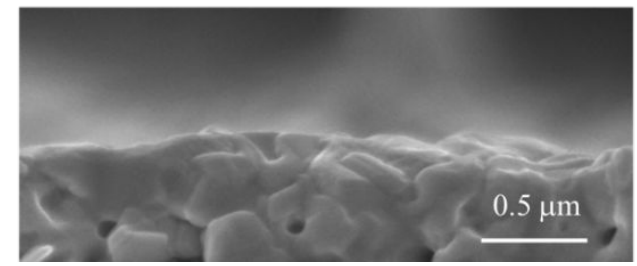

(b)

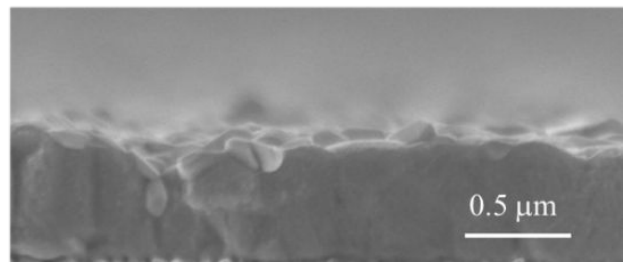

Figure S7. SEM images of (a) MA, (b) FAMA.
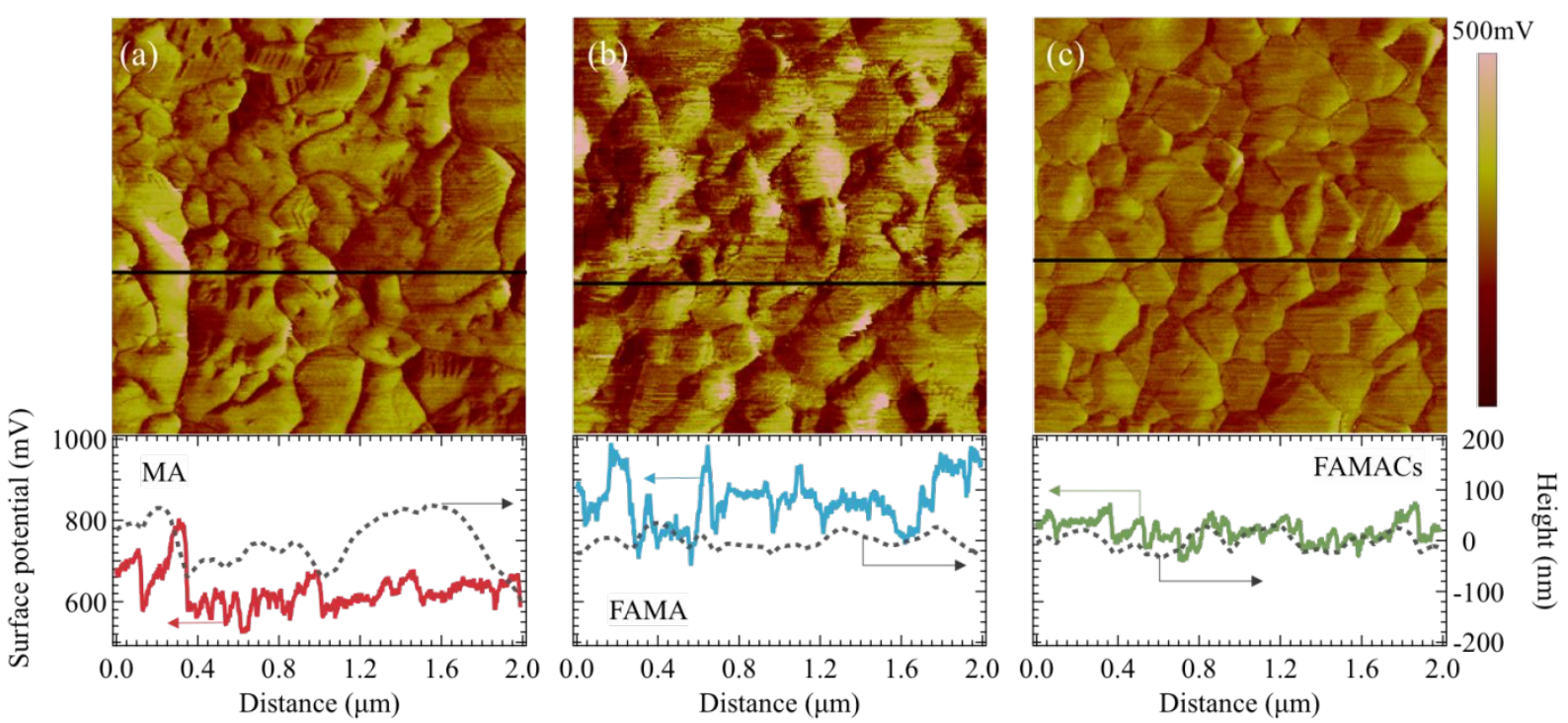

Figure S8. Surface potential and height measured by Kelvin probe force microscopy (KPFM) in lead halide perovskites. Surface potential images (upper panels) and line profiles (lower panels) in (a) MA, (b) FAMA and (c) FAMACs. Corresponding height traces are shown as the dashed lines in lower panels. 
Table S1. Best fit parameters of the total carrier recombination dynamics to $y=A_{1} e^{-\frac{x}{t_{1}}}+A_{2} e^{-\frac{x}{t_{2}}}$.

\begin{tabular}{lllll} 
Film & $\mathrm{A} 1$ & $\mathrm{t} 1(\mathrm{~ns})$ & $\mathrm{A} 2$ & $\mathrm{t} 2(\mathrm{~ns})$ \\
\hline MA & $0.690 \pm 0.005$ & $20.4 \pm 0.2$ & $0.287 \pm 0.006$ & $105 \pm 2$ \\
\hline FAMA & $0.518 \pm 0.006$ & $24.6 \pm 0.4$ & $0.436 \pm 0.006$ & $130 \pm 2$ \\
\hline FAMACs & $0.556 \pm 0.006$ & $43.3 \pm 0.5$ & $0.415 \pm 0.007$ & $201 \pm 3$
\end{tabular}

